\title{
Self-assembled monolayer of light-harvesting core complexes of photosynthetic bacteria on an amino-terminated ITO electrode
}

\author{
Yoshiharu Suemori · Morio Nagata · Yukari Nakamura · Katsunori Nakagawa • \\ Ayumi Okuda · Jun-ichi Inagaki · Kiyoshi Shinohara · Makiko Ogawa · \\ Kouji Iida · Takehisa Dewa · Keiji Yamashita - Alastair Gardiner · \\ Richard J. Cogdell · Mamoru Nango
}

Received: 8 June 2006/ Accepted: 24 August 2006/Published online: 17 November 2006

(C) Springer Science+Business Media B.V. 2006

\begin{abstract}
Light-harvesting antenna core (LH1-RC) complexes isolated from Rhodospirillum rubrum and Rhodopseudomonas palustris were successfully self-assembled on an ITO electrode modified with 3-aminopropyltriethoxysilane. Near infra-red (NIR) absorption, fluorescence, and IR spectra of these LH1RC complexes indicated that these LH1-RC complexes on the electrode were stable on the electrode. An efficient energy transfer and photocurrent responses of these LH1-RC complexes on the electrode were observed upon illumination of the LH1 complex at $880 \mathrm{~nm}$.
\end{abstract}

Keywords Light-harvesting complex $\cdot$ Light harvesting-reaction center complex $\cdot$ Photosynthesis · Electrode $\cdot$ Energy transfer $\cdot$ Photocurrent

Y. Suemori $\cdot$ M. Nagata $\cdot$ Y. Nakamura $\cdot$ K. Nakagawa

A. Okuda · J. Inagaki $\cdot$ K. Shinohara $\cdot$ M. Ogawa ·

T. Dewa $\cdot$ K. Yamashita $\cdot$ M. Nango $(\varangle)$

Department of Applied Chemistry, Tsukuri College,

Nagoya Institute of Technology, Gokiso-cho, Showa-ku,

Nagoya 466-8555 Aichi, Japan

e-mail: nango@nitech.ac.jp

K. Iida

Nagoya Municipal Industrial Research Institute,

Rokuban 3-4-41, Atsuta-ku, Nagoya 456-0058, Japan

A. Gardiner · R. J. Cogdell

Division of Biochemistry and Molecular Biology,

Institute of Biomedical and Life Sciences,

University of Glasgow, University Avenue,

Glasgow G12 8QQ, UK

\section{Introduction}

When light energy is absorbed in vivo by purple bacterial light-harvesting ( $\mathrm{LH})$ complexes it is rapidly transferred to the reaction centers (RC) where the light energy is efficiently used to drive chemical reactions (Ke 2001). In most types of purple bacteria there are two types of antenna complexes: peripheral LH2 complexes and the LH1 complexes (Ke 2001). The structure of the LH2 complex of Rhodopseudomonas acidophila strain 10050 has been resolved to a resolution of $2.0 \AA$ (McDermott et al. 1995). This LH2 complex consists of a ring of nine heterodimeric subunits. However, such high-resolution structure has not been determined for the LH1 complex yet. There are, however, low-resolution projection structures produced by transmission electron microscopy (TEM) (Karrasch et al. 1995) of two-dimensional (2D) crystals of the LH1 complex and a $4.8 \AA$ X-ray crystal structure of the LH1-RC core complex (Roszak et al. 2003). TEM analysis of the LH1 complexes revealed two types of complex, monomeric complexes from Rhodospirillum rubrum (R. rubrum) (Karrasch et al. 1995) and dimeric complexes from Rhodobacter sphaeroides ( $R$. sphaeroides) (Jungas et al. 1999). The recent crystal structure of the LH1-RC 'core' complex from Rhodopseudomonas palustris (Rps. palustris) reveals that the LH1 complex surrounds the contours of the RC so that the 'core' complex has an overall oval rather than a circular shape (Roszak et al. 2003). This structure showed the RC surrounded by the LH1 complex which consisted of 15 pairs of transmembrane helical $\alpha$ - and $\beta$-polypeptides and their coordinated BChls. The complete closure of the $\mathrm{RC}$ by the 
LH1 is prevented by a single transmembrane helix called W. Atomic force microscopy has also been used to observe antenna complexes in both natural and reconstituted membranes (Scheuring et al. 2001, 2003, 2004; Fotiadis et al. 2004; Bahatyrova et al. 2004a, b; Stamouli et al. 2003). Scheuring et al. observed the LH1 complex as a minor component together with the major LH2 complexes from Rubrivivax geratinosus (Scheuring et al. 2001) in reconstituted membranes and intact LH1-RC core complexes in native photosynthetic membranes (chromatophore) from Rhodopseudomonas viridis (Scheuring et al. 2003) and Rhodospirillum photometricum (Scheuring et al. 2004). Fotiadis et al. observed the LH1-RC core complex from $R$. rubrum in membranes formed from Escherichia coli lipids (Fotiadis et al. 2004). Bahatyr ova et al. showed that the LH1 complexes of a mutant lacking the RC from $R$. sphaeroides which form circular, elliptical, and even polygonal ring shapes as well as arcs and open rings (Bahatyrova et al. 2004b) and that the LH1 complexes are positioned to function as an energy collection hub from the LH2 complexes in native membranes (Bahatyrova et al. 2004a).

Our understanding of charge separation and energy transfer in these LH2 and LH1-RC core complexes has enabled the first steps to be taken towards generating artificial systems that convert light energy into usable electrical current. Previous attempts to produce an artificial, energy-converting electrode system used either the LH1 complexes (Ogawa et al. 2002) or RC (Blankenship et al. 1995) immobilized on the electrodes. Until now, there have only been a few attempts to immobilize intact 'core' complexes, consisting of both the LH1 and the RC components together, onto an electrode (Ogawa et al. 2004; Das et al. 2004).

We have recently developed a procedure to create a self-assembled monolayer (SAM) of reconstituted LH1 complexes on a transparent indium tin oxide (ITO) electrode modified with 3-aminopropyltriethoxysilane (APS) using electrostatic interactions or hydrogen bonding between the electrode surface and the anionic LH1 polypeptides at $\mathrm{pH}$ 8.0 (Ogawa et al. 2002). The near infra-red (NIR) absorption spectrum showed that the LH1 complex was stable when immobilized onto the electrode. Our current work extends this approach to the native LH1-RC core complexes. LH1-RC 'core' complexes isolated from $R$. rubrum and Rps. palustris were successfully assembled on an ITO electrode modified with APS (APS-ITO). Efficient energy transfer and photocurrent responses could be observed upon illumination at $880 \mathrm{~nm}$.

\section{Materials and methods}

Growth of $R$. rubrum and Rps. palustris bacterium

The photosynthetic bacteria, $R$. rubrum strain S1 and Rps. palustris strain 2.1 .6 were grown anaerobically in the light in modified Hutner's media as previously described (Roszak et al. 2003; Visschers et al. 1991).

\section{Purification of the LH1 complex of $R$. rubrum}

Chromatophores of $R$. rubrum were prepared as previously described (Visschers et al. 1991). Carotenoid was extracted from chromatophores using benzene. Usually approximately $30 \mathrm{mM}$ OG (1 mM Tris, $\mathrm{pH}$ 7.5) was added to dissolve the chromatophores until the near IR absorption band shifted from 873 to $820 \mathrm{~nm}$. Then the resultant aqueous solution was applied to a Sephadex G-100 column $(1.5 \mathrm{~cm}$ i.d. $\times$ $75 \mathrm{~cm}$ ) to separate the RC and, LH1 complexes with carotenoids and subunit $\mathrm{LH} / \mathrm{BChl}$ a complexes without carotenoid (B820 complexes). The RC was eluted immediately after the void volume and then the LH1 complexes with carotenoid. Finally the B820 complexes were collected. Absorbance $\left(\lambda_{\max }\right)$ of the LH1 complex in the absence of carotenoid $(15 \mathrm{mM}$ OG at $\left.25^{\circ} \mathrm{C}\right): 372 \mathrm{~nm}(0.86), 586$ (0.23), 870 (1.14). Absorbance $\left(\lambda_{\max }\right)$ of the LH complex in the presence of carotenoid $\left(20 \mathrm{mM} \mathrm{OG}\right.$ at $\left.25^{\circ} \mathrm{C}\right): 372 \mathrm{~nm}(0.86), 475$ (0.19), 512 (0.22), 546 (0.19), 586 (0.235), $875(0.9)$.

Isolation and purification of the core complex of $R$. rubrum and Rps. palustris

The LH1-RC core complexes isolated from $R$. rubrum and Rps. palustris were purified essentially as described previously (Roszak et al. 2003). These LH1-RC core complexes from $R$. rubrum and Rps. palustris were initially solubilized by the addition of LDAO to $0.4 \%$ or $1 \% \mathrm{v} / \mathrm{v}$ in $20 \mathrm{mM}$ Tris $-\mathrm{HCl} \mathrm{pH} \mathrm{8.0,} \mathrm{respectively.} \mathrm{The}$ LH1-RC core complex of Rps. palustris was then separated from the LH2 complexes by sucrose density centrifugation, and was further purified by ion exchange chromatography by DE52 cellulose column. The OD880 of the isolated LH1-RC core complexes was adjusted to 0.3 .

Preparation of the core complex assembled on APS-ITO electrodes

The basic methods for this have been reported previously (Ogawa et al. 2002). Transparent indium tin 
oxide electrodes were cleaned by immersion in piranha solution $\left(\mathrm{H}_{2} \mathrm{O}_{2}: \mathrm{H}_{2} \mathrm{SO}_{4}=3: 7\right)$. We obtained APS-ITO electrodes by reacting 3-aminopropyltriethoxysilane with the surface of ITO electrodes in dry benzene at $80-90^{\circ} \mathrm{C}$ for $4 \mathrm{~h}$. The OD 880 of the isolated core was adjusted to 0.3. APS-ITO electrodes were immersed in the LH complex and the core complex solutions in Tris- $\mathrm{HCl} \mathrm{pH} 8.0$ for $6 \mathrm{~h}$ at $4^{\circ} \mathrm{C}$, and then rinsed with Tris- $\mathrm{HCl} \mathrm{pH}$ 8.0. The core complexes were immobilized, as a self-assembled monolayer on an APS-ITO electrode.

\section{Near IR, FT-IR, and fluorescence spectra}

Near IR spectra were recorded with Hitachi U-2000 and U-3500. Fluorescence spectra were measured with a Nippon Roper fluorometer by using a halogen tungsten light bulb (TS-428 DC), a single monochromator (SP-150M) for selection of the excitation wavelength, a monochromator (SP-306) and a CCD detector (Spec 10-100 BR/LN) to detect the emitted fluorescence. The slits were set at $0.50 \mathrm{~mm}$ for the LH1 complexes in OG. Slits were set at $1.00 \mathrm{~mm}$ for the LH1 complex on the electrode. The samples were measured at $25^{\circ} \mathrm{C}$. FT-IR spectra were recorded with Perkin-Elmer Spectrum 2000.

\section{Photocurrent measurements}

Photocurrents were measured at $-0.2 \mathrm{~V}$ (versus $\mathrm{Ag} /$ $\mathrm{AgCl})$ in a home made cell that contained three electrodes; an APS-ITO electrode incorporating the core complex as a working electrode, an $\mathrm{Ag} / \mathrm{AgCl}$ (saturated $\mathrm{KCl}$ ) as a reference electrode, and a platinum flake as a counter electrode. The working electrode was illuminated with a halogen lamp unit, AT-100HG, through a monochromator, SPG-120S (Shimadzu). The solution consisted of $0.1 \mathrm{M}$ phosphate buffer ( $\mathrm{pH} 7.0$ ), containing $0.1 \mathrm{M} \mathrm{NaClO}_{4}$ and $5 \mathrm{mM}$ methyl viologen.

\section{Results and discussion}

Figure 1 shows the NIR absorption spectra of the isolated R. rubrum (a) and Rps. palustris (b) core complexes in $20 \mathrm{mM}$ Tris- $\mathrm{HCl}$ buffer $\mathrm{pH}$ 8.0 OG micelle (dotted line) and assembled onto an APS-ITO electrode (solid line), respectively. These spectra show that these core complexes have the absorption maximum at $880 \mathrm{~nm}$ with two smaller peaks at 800 and $760 \mathrm{~nm}$. The former peak is attributable to the overlap of bacteriochlorophyll $a(\mathrm{BChl} a)$ in the LH1 complex $(880 \mathrm{~nm})$ and the reaction center BChl $a$ dimer 'special pair' $(870 \mathrm{~nm})$ and the latter two peaks to the BChla called 'accessory' $(800 \mathrm{~nm})$ and bacteriopheophytin $(760 \mathrm{~nm})$ in the RC, respectively (Blankenship et al. 1995). The NIR absorption spectra of these core complex on the electrode indicate that these complexes were not denatured when assembled onto an APS-ITO. In the previous study it was apparent that when the RC of $R$. rubrum was assembled, by itself, on the electrode it was relatively labile (Matsumoto et al. 1999). In present study, the complete core complex proved to be quite stable, when assembled onto the electrode. The enhanced stability of the RC surrounded by the LH1 complex probably results from supportive interactions between the two complexes.

Table 1 shows the NIR absorption and fluorescence bands of the LH1 complex of $R$. rubrum and the LH1RC core complexes of $R$. rubrum and Rps. palustris in OG micelles and on an APS-ITO electrode. The fluorescence bands of these core complexes on the electrode are identical to those in the OG micelles, again indicating that these complexes are stable when assembled onto the APS-ITO electrode. Interestingly, when illuminating at $880 \mathrm{~nm}$ the fluorescence emission of BChla molecules in the LH complex of $R$. rubrum on the APS-ITO was strongly quenched, due to the presence of the RC of $R$. rubrum. This indicates that an efficient energy transfer from BChl $a$ in the LH1 complex to the RC in the core complex is still occurring on the electrode (data not shown) (Ogawa et al. 2004).
Fig. 1 NIR absorption spectra of the isolated $R$. rubrum (a) and Rps. palustris (b) core complexes in $20 \mathrm{mM}$ Tris- $\mathrm{HCl}$ buffer $\mathrm{pH}$ 8.0 OG micelle (dotted line) and assembled onto an APS-ITO electrode (solid line)
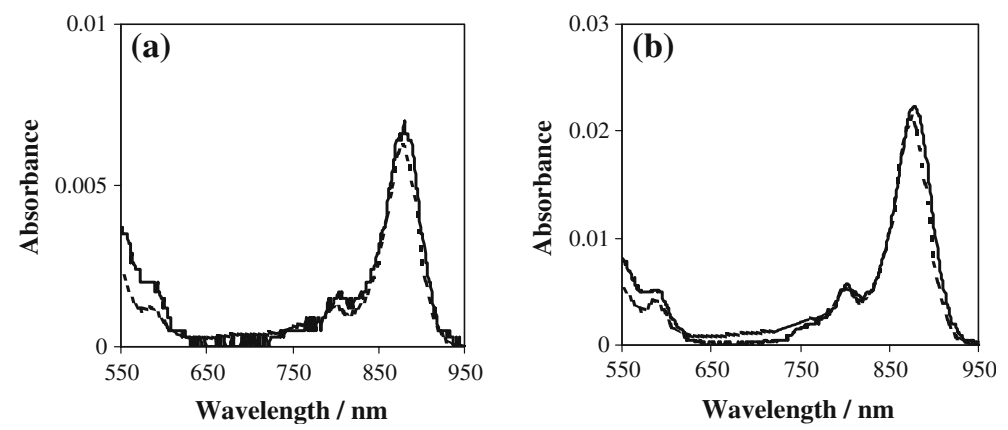
Table 1 NIR absorption and fluorescence bands of the LH1 complex of $R$. rubrum and the LH1-RC core complexes of $R$. rubrum and Rps. palustris

\begin{tabular}{lllllll}
\hline & & \multicolumn{2}{l}{ In OG micelle } & & \multicolumn{2}{l}{ On APS-ITO } \\
\cline { 3 - 4 } & & $\begin{array}{l}\text { Absorbance } \\
(\mathrm{nm})\end{array}$ & $\begin{array}{l}\text { Fluorescence } \\
(\mathrm{nm})\end{array}$ & & $\begin{array}{l}\text { Absorbance } \\
(\mathrm{nm})\end{array}$ & $\begin{array}{l}\text { Fluorescence } \\
(\mathrm{nm})\end{array}$ \\
\hline R. rubum & LH1 & 870 & 890 & 870 & 890 \\
R. rubum & LH1-RC & 880 & 900 & & 880 & 900 \\
Rps. palustris & LH1-RC & 878 & 900 & & 878 & 900 \\
\hline
\end{tabular}

FT-IR spectra of the LH complex of $R$. rubrum and the LH1-RC core complexes of R. rubrum and Rps. palustris assembled on the APS-ITO show the absorptions at 1650 and $1550 \mathrm{~cm}^{-1}$. These bands can be assigned to the amide I ( $\mathrm{C}=\mathrm{O}$ stretching vibration $)$ and amide II $(\mathrm{N}-\mathrm{H}$ deformation vibration) bands of $\alpha$ helical conformation, respectively (Miura et al. 1998). These results indicate that the LH polypeptides are in the same $\alpha$ helical configurations on the ITO electrode as in OG micelles (Parkes-Loach et al. 1988; Kashiwada et al. 2000).

In summary, all of these results indicate that the LH1 and LH1-RC core complexes were not denatured by binding to the APS-ITO surface. Similar results were obtained using Langmuir-Blodgett (LB) films to lay down LH1 complex membranes as a layer on a glass substrate (Iida et al. 2000). The LH1 complex of $R$. rubrum and the LH1-RC core complexes of $R$. rubrum and Rps. palustris on the APS-ITO electrode were stable enough to handle at $4^{\circ} \mathrm{C}$ in the dark condition least $24 \mathrm{~h}$.

Figure 2 shows the time course of the photocurrent generated from the LH1-RC core complex, LH1 complex or the RC of $R$. rubrum assembled onto an APS-ITO when the electrode was illuminated with a

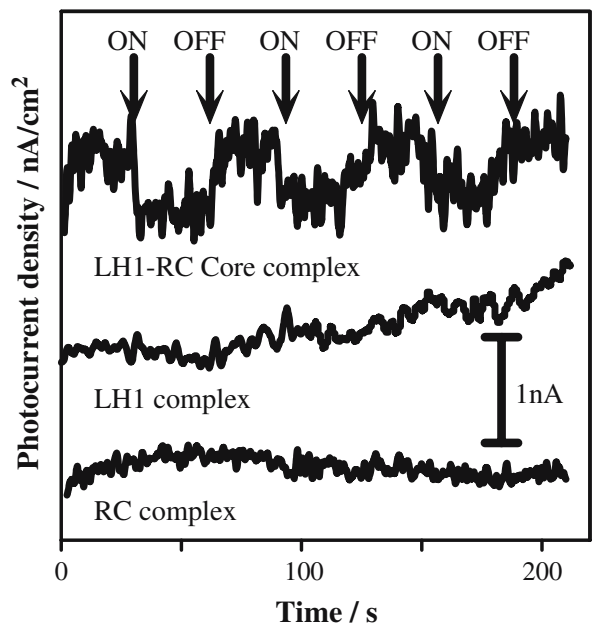

Fig. 2 Time course of the photocurrent of the LH1-RC core complex, LH1 complex or the RC complex of R. rubrum on an APS-ITO electrode when the electrode is illuminated with pulsed light $(880 \mathrm{~nm})$ firing continuously for $30 \mathrm{~s}$ pulse of light at $880 \mathrm{~nm}$. It is clear in Fig. 2 that an enhanced photocurrent was observed for the LH1-RC core complex. In contrast no photocurrent was observed for either LH1 complex or the RC. Under our experimental conditions a cathodic photocurrent was observed, implying that one-way electron transfer from pigments in the LH1-RC core complex to methyl viologen was occurring as shown in Fig. 3 (Nagata et al. 2003; Imahori et al. 2000).

Figure 4 shows excitation spectrum of the photocurrent density (dots) and the NIR absorption spectra (solid line) from R. rubrum (a) and Rps. palustris (b) LH1-RC core complexes assembled onto an APS-ITO, respectively. These photocurrent responses showed a maximum at the wavelength corresponding to the absorption bands of the complex. Interestingly, an enhanced photocurrent was observed especially upon illumination at $880 \mathrm{~nm}$ for both R. rubrum (a) and Rps. palustris (b) LH1-RC core complexes. The quantum yield of the photocurrent was $0.05 \%$ for both the $R$. rubrum and Rps. palustris complexes (Imahori et al. 2000). When the LH1 complex of $R$. rubrum alone, was immobilized on the electrode, the observed photocurrent was mainly generated by light absorbed at $770 \mathrm{~nm}$, i.e. from monomeric BChla (Nagata et al. 2003). Furthermore, when the RC complex of $R$. rubrum only was immobilized on the electrode, an efficient photocurrent was not observed upon illumination at $880 \mathrm{~nm}$ as shown in Fig. 2. Thus, the enhanced photocurrent observed at $880 \mathrm{~nm}$ in the assembled LH1-RC core complex can be ascribed to energy transfer from the LH1 to the RC and then electron transfer from the electrode to the RC as shown in Fig. 3 (Ogawa et al. 2004). This data indicates that the LH1-RC core complex was well organized on the ITO and the photocurrents were driven by light that was initially absorbed by the LH components.

In conclusion, the SAM method is clearly successful in allowing assembly of functional LH1-RC core complexes on the electrode. This has been confirmed by NIR absorption spectroscopy, demonstrating that the photocurrent response, which is derived from electron transfer between the RC and the electrode, is enhanced by illumination at $880 \mathrm{~nm}$. 


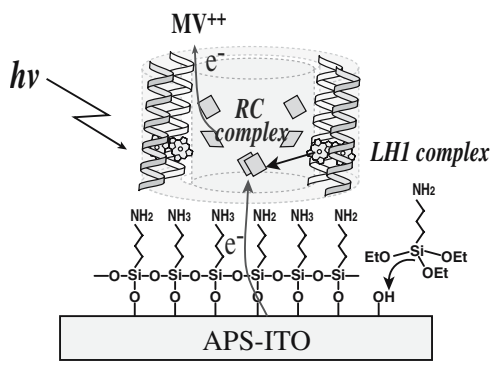

Fig. 3 Schematic drawing of LH1-RC core complexes on an APS-ITO electrode generated cathodic photocurrent which shows the electron flow from the complex to methyl viologen

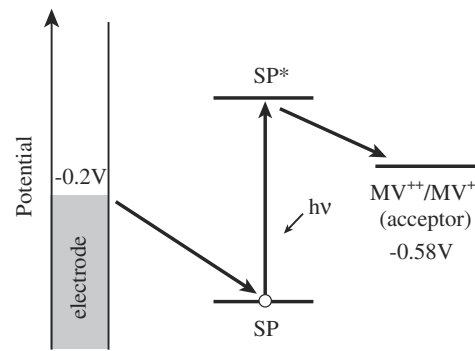

(left) according to the cathodic photocurrent as shown in Fig. 2. Energy diagram for cathodic photocurrent generation by the LH1-RC core complex (right)
Fig. 4 Photocurrent density (dots) and NIR absorption spectrum (solid line) of LH1$\mathrm{RC}$ core complexes form: (a) R. rubrum and (b) Rps. palustris assembled on an APS-ITO electrode

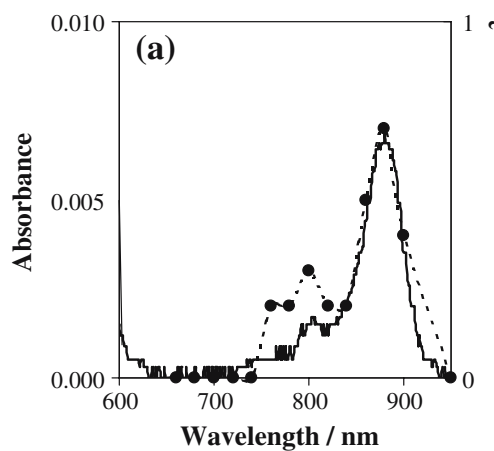

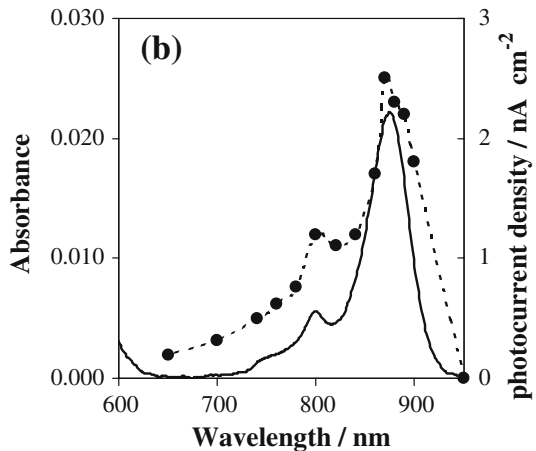

Acknowledgments M.N. and R.J.C. are grateful to the international joint grant of NEDO, BBSRC and a Japan Partnering Award for financial support. The present work was partially supported by a Grant-in-Aid for Scientific Research and Priority Area (417) from the Ministry of Education, Culture, Sports, Science and Technology (MEXT) of Japanese Government.

\section{References}

Bahatyrova S, Frese RN, Siebert CA, Olsen JD, van der Werf K, van Grondelle R, Niederman RA, BulloghPA, Otto C, Hunter CN (2004) Nature 430:1058-1062

Bahatyrova S, Frese RN, van der Werf KO, Otto C, Hunter CN, Olsen JD (2004) J Biol Chem 279:21327-21333

Blankenship RE, Madigan MT, Bauer CE (1995) Anoxygenic photosynthetic bacteria. Kluwer Academic Publishers, Dordrecht

Das R, Kiley PJ, Segal M, Norville J, Yu AA, Wang L, Trammell SA, Reddick LE, Kumar R, Stellacci F Lebedev N, Schnur J, Bruce BD, Zhang S, Baldo M (2004) Nano Lett 4(6):1079-1083

Fotiadis D, Qian P, Philippsen A, Bullough PA, Engel A, Hunter CN (2004) J Biol Chem 279:2063-2068

Iida K, Kashiwada A, Nango M (2000) Colloids Surf A 169:199_ 208

Imahori H, Yamada H, Nishimura Y, Yamazaki I, Sakata Y (2000) J Phys Chem B 104:2099-2108

Jungas C, Ranck J-L, Joliot P, Vermeglio A (1999) EMBO J 18:534-542

Karrasch S, Bullough P, Ghosh R (1995) EMBO J 14:631-638

Kashiwada A, Watanabe H, Tanaka T, Nango M (2000) Chem Lett 24-25
Ke B (2001) Photosynthesis, in Govinjee. Kluwer Academic Publishers, Dordrecht

Matsumoto K, Nomura K, Tohnai Y, Fujioka S, Wada M, Erabi T (1999) Bull Chem Soc Jpn 72:2169

McDermott G, Prince SM, Freer AA, Hawthornthwaite-Lawless AM, Papiz MZ, Cogdell RJ, Isaacs NW (1995) Nature 374:517-521

Miura Y, Kimura S, Imanishi Y, Umemura J (1998) Langmuir 14:6935-6940

Nagata M, Yoshimura Y, Inagaki J, Suemori Y, Iida K, Ohtsuka T, Nango M (2003) Chem Lett 32:852-853

Ogawa M, Kanda R, Dewa T, Iida K, Nango M (2002) Chem Lett 31:464-465

Ogawa M, Shinohara K, Nakamura Y, Suemori Y, Nagata M, Iida K, Gardiner AT, Cogdell RJ, Nango M (2004) Chem Lett 33:772-773

Parkes-Loach S, Sprinkle JR, Loach PA (1988) Biochemistry 27:2718

Roszak AW, Howard TD, Southall J, Gardiner A, LawCJ, Isaacs NW, Cogdell RJ (2003) Science 302:1969-1972

Scheuring S, Reiss-Husson F, Engel A, Rigaud J-L, Ranck J-L (2001) EMBO J 20:3029-3035

Scheuring S, Seguin J, Marco S, Levy D, Bruno R, Rigaud J-L (2003) Proc Natl Acad Sci USA 100:1690-1693

Scheuring S, Sturgis JN, Prima V, Bernadac A, Lèvy D, Rigaud J-L (2004) Proc Natl Acad Sci USA 101:11293-11297

Stamouli A, Kafi S, Klein DCG, Oosterkamp TH, Frenken JWM, Cogdell RJ, Aartsma TJ (2003) Biophys J 84:24832491

Visschers RW, Chang MC, van Mourik F, Parkes-Loach PS, Heller BA, Loach PA, van Grondelle R, (1991) Biochemistry 30:5734-5742 